\title{
BMJ Open Effectiveness of pharmacological treatments in Duchenne muscular dystrophy: a protocol for a systematic review and meta-analysis
}

\author{
Carlos Pascual Morena, ${ }^{1}$ Vicente Martinez-Vizcaino, ${ }^{1,2}$ Celia Álvarez-Bueno, ${ }^{\oplus, 3}$ \\ Ruben Fernández Rodríguez, ${ }^{1}$ Estela Jiménez López, ${ }^{1,4}$ \\ Ana Isabel Torres-Costoso, ${ }^{5}$ Iván Cavero-Redondo ${ }^{1,3}$
}

To cite: Pascual Morena C, Martinez-Vizcaino V, ÁlvarezBueno $\mathrm{C}$, et al. Effectiveness of pharmacological treatments in Duchenne muscular dystrophy: a protocol for a systematic review and meta-analysis. BMJ Open 2019;9:e029341. doi:10.1136/ bmjopen-2019-029341

- Prepublication history for this paper is available online. To view these files please visit the journal online (http://dx.doi. org/10.1136/bmjopen-2019029341).

Received 22 January 2019 Revised 16 August 2019 Accepted 22 August 2019
Check for updates

(c) Author(s) (or their employer(s)) 2019. Re-use permitted under CC BY-NC. No commercial re-use. See rights and permissions. Published by BMJ.

For numbered affiliations see end of article.

Correspondence to Professor Vicente MartinezVizcaino;

Vicente.Martinez@uclm.es

\section{ABSTRACT}

Introduction In recent years, important advances have been made in the treatment of Duchenne muscular dystrophy (DMD). This protocol proposes a methodology for carrying out a systematic review and meta-analysis that aims to: (1) improve the evidence of the benefits of different pharmacological treatments in boys with DMD, and (2) compare the benefit of treatments specifically aimed at delaying the progression of disease in the functional outcomes.

Methods and analysis This protocol is guided by the Preferred Reporting Items for Systematic Review and Meta-Analysis Protocols (PRISMA-P) and by the Cochrane Collaboration Handbook. A thorough selection of the literature will be done through the MEDLINE, EMBASE and Web of Science databases. The search will be conducted in English and Spanish. The Risk of Bias 2.0 tool from the Cochrane Collaboration will be used to assess the risk of bias. A narrative synthesis of the data will be performed. Meta-analysis will be conducted for effect of treatment on the 6 min walking distance (6MWD), North Star Ambulatory Assessment and Timed Functional Tests. Subgroup analyses will be performed by age or baseline values of the 6MWD, and overall bias.

Ethics and dissemination The approval of an ethical committee is not required. All the included trials will comply with the current ethical standards and the Declaration of Helsinki. The results of this proposed systematic review and meta-analysis will provide a general overview and evidence concerning the effectiveness of pharmacological treatments in Duchenne muscular dystrophy. Findings will be disseminated to academic audiences through peer-reviewed publications, as well as to clinical audiences, patients' associations and policy makers, and may influence guideline developers in order to improve outcomes for these patients.

PROSPERO registration number CRD42018102207

\section{INTRODUCTION}

\section{Background}

Duchenne muscular dystrophy (DMD) is a severe X-linked recessive disease, ${ }^{1}$ with an incidence of 1 per 3500-9000 male children born alive, ${ }^{2}$ although exceptionally, some
Strengths and limitations of this study

- This study presents an explicit and replicable methodology to analyse the effect of novel drug treatments in Duchenne muscular dystrophy on functional, histological and biochemical outcomes in order to provide clinicians with a comprehensive synthesis of the evidence on the current pharmacological strategies for this disease, identifying potential risk of bias.

- Two researchers will independently perform study selection, data extraction and quality assessment.

- The assessment of risk of bias of the selected studies and heterogeneity among studies included, with particular reference to sample characteristics, is a featured point in this evidence review.

- The differences among novel drug treatments might be a source of variable quality and heterogeneity among studies and may limit the quality of the evidence of this meta-analysis.

cases have been observed in girls. ${ }^{3}$ In DMD, the $D M D$ gene (which encodes the protein dystrophin) is affected by point mutations, deletions and duplications of parts of the gene, causing alterations in the reading frame and truncation of the dystrophin protein, which is rapidly degraded. Due to the almost total absence of dystrophin, muscle tissue degenerates and is replaced by fibrotic tissue due to the body's inability to repair the muscle damage caused. Typically, a DMD diagnosis occurs in early childhood through genetic testing, biochemical tests and muscle biopsy. In the first years of life, the child gains strength and motor skills, although less than peers. Later, affected children progressively deteriorate, with loss of muscular strength and ability to ambulate. Other typical complications of DMD include scoliosis, heart failure, respiratory insufficiency, ${ }^{1}$ fractures of long bones and vertebrae due to osteoporosis ${ }^{4}$ 
and eventually death. ${ }^{1}$ However, improvements in the management of DMD have delayed the onset of these events. The use of glucocorticoids and physiotherapy delays the loss of strength and ambulation ${ }^{56}$; early pharmacological therapy for heart failure improves prognosis and survival ${ }^{7}$; and surgery for scoliosis, respiratory physiotherapy and the use of non-invasive ventilation have enhanced lung capacity, hypercapnia and respiratory failure, increasing survival. ${ }^{8-10}$ Also, a high interindividual intrinsic variability in the progression of the disease due to the type of mutation in $D M D$, or to polymorphisms in other genes, is characteristic. ${ }^{11}$

\section{State of the art}

Because of the lack of effective treatments, several trials of potential new treatments are being carried out. Some are still in the preclinical experimentation phase, but others are already in the clinical phase, including some which are approved by certain drug agencies. ${ }^{612} 13$ These studies are mostly focused on the partial production of dystrophin, as in Becker muscular dystrophy (BMD), which has a less severe prognosis than DMD because the reading frame of the gene is not affected, and a partially functional dystrophin is produced. ${ }^{14}$ Almost two decades ago, gentamicin was found to cause in a small percentage of patients some production of dystrophin by forcing the reading of premature stop codons with other tRNAs and being able to continue the synthesis of protein. ${ }^{15}$ Ataluren, with the same mechanism of action, has shown more efficacy than gentamicin at promoting nonsense readthrough. ${ }^{16}$ Furthermore, another treatment for a different subgroup of DMD patients is based on the omission of exon 51 to recover the reading frame. This mechanism is used by eteplirsen and drisapersen. ${ }^{17}$ Additionally, another line of research is based on reversing muscle fibrosis in the absence of dystrophin by epigenetic modifications, such as givinostat. ${ }^{18}$ Finally, there are also lines of research aimed at examining the production of utrophin, a paralog of dystrophin ${ }^{19}$ and the omission of other exons. ${ }^{20}$

In recent years, the interest in the potential of gene editing has increased, since new therapeutic strategies such as inserting dystrophin functional genes through an adenovirus, ${ }^{21}$ repairing the affected gene using the CRISPR/Cas9 technique ${ }^{22}$ or transplanting muscle stem cells to the affected muscles ${ }^{22} 23$ have proven effective.

Despite numerous trials testing new treatments for DMD, there is a lack of evidence about the effectiveness of these treatments on delaying the progression of the disease, as well as the conditioning factors that could modify this effectiveness, such as the baseline characteristics of patients or their age.

\section{Objectives}

This protocol proposes a methodology for carrying out a systematic review and meta-analysis that aims to: (1) improve evidence of the benefits of different pharmacological treatments in boys with DMD in the main functional, histological and biochemical outcomes that characterise the disease and its complications, through systematic review; and (2) to compare, through meta-analysis, the benefit of treatments specifically aimed at delaying the progression of disease on functional outcomes $(6 \mathrm{~min}$ walking distance [6MWD], North Star Ambulatory Assessment [NSAA] and Timed Functional Tests [TFT]).

\section{Methodology}

This systematic review and meta-analysis has been registered in the PROSPERO database (registration number: CRD42018102207) and will be conducted according to the Preferred Reporting Items for Systematic review and Meta-Analysis Protocols (PRISMA-P) and The Cochrane Handbook for Systematic Reviews of Interventions.

\section{Inclusion/exclusion criteria for the selection of studies}

Type of study design: Clinical trials without restriction based on allocation or blinding strategy.

Type of participants: Clinical trials which have included boys with confirmed DMD. Trials with more classes of patients will also be included, when data for DMD patients is provided separately.

Type of interventions: (1) Specific medications for DMD, (2) non-specific medications used in DMD, (3) gene therapy. There will be no restriction on the duration of the trials nor on the associated use of glucocorticoids or other treatments (although it is foreseeable that for each trial the researcher will impose more restrictive criteria).

Type of outcome: Change in one or several of the following tests: (1) 6MWD will be the main outcome, in which the space walked by the boy during $6 \mathrm{~min}$ is determined, expressed in metres; (2) other functional tests: North Star Ambulatory Assessment, manual muscle test, 2 min walking distance, timed functional test; (3) cardiorespiratory function: change in forced vital capacity, maximum expiratory pressure, maximum inspiratory pressure, ejection volume, cardiac output and electrocardiographic parameters; (4) histological changes: change in dystrophin expression, change in the number of positive fibres to dystrophin, absolute or proportional change in muscle and fibrotic tissue; (5) molecular changes: detection of omission of exon 51 by reverse transcription polymerase chain reaction (RT-PCR); (6) change in biochemical tests: creatine kinase (CK), lactate dehydrogenase (LDH) levels and proinflammatory cytokines.

\section{Search method for the identification of trials \\ Electronic search}

MEDLINE (via PubMed), EMBASE and Web of Science databases will be used. A search will be conducted from inception until 31 July 2019. Mendeley will be used as a reference manager. Searches for unpublished studies will be conducted at: OPEN GRAY, ProQuest dissertations \& Thesis Global, Theseo, Networked Digital Library of Theses and Dissertations (NDLTD) and Google Scholar. A search of ClinicalTrials.gov and EudraCT clinical 
trial records will also be conducted. The search will be conducted in English and Spanish.

The search strategy terms will be as follows: ('dystrophy' OR 'duchenne' OR 'dmd' OR 'dystrophinopathy') AND ('gentamicin' OR 'ataluren' OR 'ptc124' OR 'eteplirsen' OR 'avi-4658' OR 'drisapersen' OR 'pro051' OR 'golodirsen' OR 'casimersen' OR 'antisense oligonucleotides' OR 'exon skipping') AND ('trial' OR 'randomized clinical trial' OR 'randomized controlled trial' OR 'clinical trial').

\section{Selection and analysis of trials}

Following the exclusion of duplicates, the titles and summaries of each study will be evaluated in a first phase for inclusion or exclusion. Articles that do not provide enough information in the title or abstract will also be included. When necessary, the corresponding authors of the potentially included studies will be contacted to obtain any missing information. In a second phase, the selected studies will be fully examined to identify the studies that will finally be included in the systematic review. This process will be outlined in a flowchart, as detailed in the PRISMA guidelines. ${ }^{24}$ Both phases of the selection of studies will be conducted separately by two independent reviewers. The reviewers will not be blinded to authors, institutions or journals. Any disagreements will be resolved by consensus and, if necessary, by a third reviewer.

The following information will be individually extracted from the selected articles by the same two reviewers: author, year of publication, country, drug, sample, number of participants in each intervention group, age, phase of the study, design, duration, dosage and outcome (table 1). In the summary of the demographic data in the 'Results' section, the duplication of the number of patients will be avoided if there are studies that use the same sample. Disagreements will be resolved by consensus or, if necessary, by a third reviewer.

\section{Assessment of the quality of the studies: risk of bias}

The risk of bias assessment will be conducted using the RoB 2.0 tool developed by the Cochrane Collaboration. This version includes five domains: bias arising from the randomisation process, bias due to deviations from intended interventions, bias due to missing outcome data, bias in measurement of the outcome, and bias in selection of the reported result. Each domain is scored as high risk, some concerns or low risk. A sixth domain, overall bias, will have a low risk result if the rest of the domains are of low risk, some concerns if there are some domains assessed with some concerns; and high risk if there are one or more domains with high risk of bias. Studies scoring as high risk will be removed from the meta-analysis.

\section{Data synthesis}

Forest plots will be used to indicate the effect size, with $95 \%$ confidence interval (CI) for the effect of interventions on 
the change in 6MWD (measured as difference in metres \pm standard deviation $[\mathrm{SD}]$ ), the change in TFT (up four stairs, down four stairs, run 10 metres, supine to stand; measured in seconds $\pm \mathrm{SD}$ ) and the change in $\mathrm{NSAA} \pm \mathrm{SD}$ (table 2). In conducting each meta-analysis, at least five trials that determine the change in the outcome for a specific drug will be needed. All trials included in the meta-analyses will be controlled trials, compared with placebo, non-intervention and matched historical cohort. In cross-over trials, we will preferably use the results at the end of the first period. A narrative synthesis of the data will be presented for the remaining outcomes.

To compare the effect of the different drug treatments, a pairwise meta-analysis for direct and indirect comparisons between drug treatment and placebo or non-intervention will be carried out. The meta-analysis will be conducted using STATA V.15, combining the differences of means and their corresponding 95\% CI. Fixed-effects or random-effects models will be used, depending on the heterogeneity. The heterogeneity of results across studies will be evaluated using the $\mathrm{I}^{2}$ statistic, which is considered as: $<40 \%$ low; $30 \%-60 \%$ substantial; $50 \%-90 \%$ significant; and $>75 \%$ considerable. The $\mathrm{p}$ values will be considered.

Publication bias will be assessed using a funnel plot, which contrasts the measure of effect size against its standard error (SE). The asymmetry of the graph will be checked visually. Additionally, the Egger method will be used with a $\mathrm{p}<0.10$ indicating risk of publication bias. The strength of the evidence will be assessed using the GRADE tool of the Cochrane library.

\section{Analysis by subgroups}

Outcomes by sample subgroups (ie, by age or baseline values of the 6MWD) will be included in the narrative and forest plot synthesis. When two or more measures taken in the same way and for the same subgroup of patients are available, the corresponding meta-analysis will be performed, using the same criteria as those previously described.

In addition, a subgroup analysis will be conducted depending on the result obtained in the assessment of the risk of bias. Thus, two subgroups will be considered: without domains with a high risk of bias; and with one or more domains with a high risk of bias.

\section{Sensitivity analysis}

If a meta-analysis is performed, a sensitivity analysis will be conducted, excluding studies from the analysis one by one. These will be performed to examine the potential influence of each study in the pooled estimates.

\section{Study status}

Currently, preliminary searches piloting the study selection strategy, eligibility criteria and data extraction procedures have been performed. The design of the final search strategy and data extraction form will be developed based on preliminary findings. Formal data search on databases and study selection will be conducted on 31 July 2019.

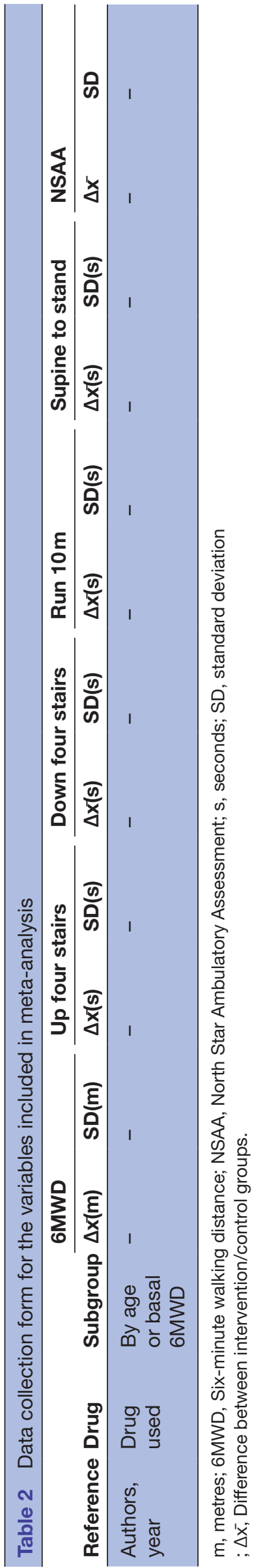


Then, the quality assessment and final data analysis will be performed.

\section{Patient and public involvement}

Patients and the public were not involved in the design and conception of this study.

\section{Ethical considerations}

The approval of an ethical committee is not required to conduct this study. All included trials will be in accordance with the Declaration of Helsinki and current ethical standards. Results of this systematic review and meta-analysis will provide a general synthesis of evidence concerning the effectiveness of pharmacological treatments in DMD. On completion of this study, a dissemination plan will be conducted, which includes (1) presenting findings of this study at international neurology-related conferences; (2) publishing our results in peer-reviewed high-impact academic journals; and (3) to send briefing notes to social media in order to influence decision makers and guideline developers.

\section{DISCUSSION}

There are currently several ongoing clinical trials and many others completed for DMD. These trials are divided into those that use existing drugs, which can improve the available evidence, and those that introduce new drugs with a potential benefit in DMD, whether specific to the disease or not. For this reason, given the relevance of the disease as well as the need for a periodic update of knowledge about the treatment of DMD, this systematic review and meta-analysis has been proposed.

We expect to provide the following results. First, we will show the effectiveness of different drugs in slowing down the disease, using the 6MWD as the main outcome. Progressive and accelerated loss of the ability to walk is the main characteristic of DMD and delaying this progression could be the best indicator of the effectiveness of a treatment. The 6MWD is a very simple and inexpensive test. ${ }^{25}$ Biochemical tests, such as CK levels, are deceptive in advanced stages of the disease. It is known that when there is a significant loss of muscle mass, CK decreases ${ }^{26}$; however, the results of these secondary tests will be included whenever available. Likewise, in addition to the 6MWD analysis, the results of the meta-analysis for TFT and NSAA could reinforce the evidence on the efficacy of these treatments. Although the change in $6 \mathrm{MWD}$ is the main outcome used in clinical trials, changes in TFT and NSAA are associated with the progression of the disease.

In addition, regarding 6MWD, two stages are shown in the disease: the first stage with an improvement of $6 \mathrm{MWD}$ up to the age of 10 years old, and a second stage with a progressive worsening until the loss of ambulation and wheelchair dependence. ${ }^{25}$ For this reason, stratification of patients over and under 7 years has been proposed in clinical trials. It is likely that an effective treatment will show a greater effect in patients with greater decline.
However, the greatest clinical benefit is expected in early stages of the disease. ${ }^{27}$

Several concerns must be considered in the future analysis. Ataluren and gentamicin have a different pharmacological mechanism of action than that of eteplirsen and drisapersen. Therefore, they are targeted to different subgroups of patients and they cannot be exchanged with one another, and only the efficacy of drugs with the same target population can be compared, that is, gentamicin versus ataluren and eteplirsen versus drisapersen In particular, this last case can shed light on why eteplirsen is approved by the FDA, ${ }^{12}$ unlike drisapersen, ${ }^{28}$ and whether that authorisation is justified. In contrast, the comparison of drugs with different mechanisms of action will indicate which subgroup of patients can potentially benefit more.

We also expect to update existing evidence with data from upcoming trials using new pharmacological therapies. This is an important factor, as the improvement in survival and quality of life in recent decades has been due to better management in associated complications, especially respiratory and cardiac. Its optimisation, through comparing certain pharmacological groups to others, or of one drug to another within the same group, can provide a better prognosis for these patients.

\section{Limitations}

This review will have potential limitations such as those related with the literature search strategy, since some potentially relevant documents ( $\mathrm{PhD}$ dissertations, abstract of symposiums, etc) might be missed and undermine the sensitivity of the search strategy. Additionally, main multidisciplinary databases (proposed by the Cochrane Handbook for Systematic Reviews of Interventions) will be searched but some bias could be produced as the searching of specific databases is not planned. Additionally, due to the lack of data in some outcomes, a quantitative analysis may not be possible. To minimise these limitations, PRISMA and the Cochrane Handbook for Systematic Reviews of Interventions recommendations will be used.

\section{Author affiliations}

${ }^{1}$ Universidad de Castilla-La Mancha, Health and Social Research Center, Cuenca, Spain

${ }^{2}$ Universidad Autónoma de Chile, Facultad de Ciencias de la Salud, Talca, Chile ${ }^{3}$ Universidad Politecnica y Artísitca del Paraguay, Asunción, Paraguay ${ }^{4}$ CIBERSAM (Biomedical Research Networking Centre in Mental Health), Madrid, Spain

${ }^{5}$ School of Nursing and Physiotherapy, Universidad de Castilla-La Mancha, Toledo, Spain

Contributors CP-M and IC-R designed the study. VM-V was the principal investigator and guarantor. CP-M and IC-R were the main coordinators of the study. CP-M, CA-B and VM-V conducted the study. IC-R, CA-B, RF-R, EJ-L and AIT-C gave statistical and epidemiological support. CP-M wrote the article with the support of IC-R and CA-B. All authors revised and approved the final version of the manuscript.

Funding This study was funded by Apadrina la Ciencia. CP-M is supported by a grant from the Universidad de Castilla-La Mancha (2018-CPUCLM-7939).

Competing interests None declared.

Patient consent for publication Not required. 
Provenance and peer review Not commissioned; externally peer reviewed.

Open access This is an open access article distributed in accordance with the Creative Commons Attribution Non Commercial (CC BY-NC 4.0) license, which permits others to distribute, remix, adapt, build upon this work non-commercially, and license their derivative works on different terms, provided the original work is properly cited, appropriate credit is given, any changes made indicated, and the use is non-commercial. See: http://creativecommons.org/licenses/by-nc/4.0/.

\section{REFERENCES}

1. Yiu EM, Kornberg AJ, dystrophy Dmuscular. Duchenne muscular dystrophy. J Paediatr Child Health 2015;51:759-64.

2. Mah JK, Korngut L, Dykeman J, et al. A systematic review and metaanalysis on the epidemiology of Duchenne and Becker muscular dystrophy. Neuromuscular Disorders 2014;24:482-91.

3. Giliberto F, Radic CP, Luce L, et al. Symptomatic female carriers of Duchenne muscular dystrophy (DMD): genetic and clinical characterization. J Neurol Sci 2014;336:36-41.

4. Ward LM, Hadjiyannakis S, McMillan HJ, et al. Bone health and osteoporosis management of the patient with Duchenne muscular dystrophy. Pediatrics 2018;142(Supplement 2):S34-S42.

5. McDonald CM, Henricson EK, Abresch RT, et al. Long-Term effects of glucocorticoids on function, quality of life, and survival in patients with Duchenne muscular dystrophy: a prospective cohort study. The Lancet 2018;391:451-61.

6. Birnkrant DJ, Bushby K, Bann CM, et al. Diagnosis and management of Duchenne muscular dystrophy, part 1: diagnosis, and neuromuscular, rehabilitation, endocrine, and gastrointestinal and nutritional management. The Lancet Neurology 2018;17:251-67.

7. Buddhe S, Cripe L, Friedland-Little J, et al. Cardiac management of the patient with Duchenne muscular dystrophy. Pediatrics 2018;142(Supplement 2):S72-S81.

8. Birnkrant DJ, Bushby K, Bann CM, et al. Diagnosis and management of Duchenne muscular dystrophy, part 2: respiratory, cardiac, bone health, and orthopaedic management. Lancet Neurol 2018;17:347-61.

9. Cheuk DKL, Wong V, Wraige E, et al. Surgery for scoliosis in Duchenne muscular dystrophy. Cochrane Database Syst Rev $2015 ; 22$.

10. Sheehan DW, Birnkrant DJ, Benditt JO, et al. Respiratory management of the patient with Duchenne muscular dystrophy. Pediatrics 2018;142(Supplement 2):S62-S71.

11. Barakat-Haddad $\mathrm{C}$, Shin S, Candundo H, et al. A systematic review of risk factors associated with muscular dystrophies. Neurotoxicology 2017;61:55-62.

12. Aartsma-Rus A, Krieg AM. Fda Approves Eteplirsen for Duchenne muscular dystrophy: the next chapter in the Eteplirsen SAGA. Nucleic Acid Ther 2017;27:1-3.

13. Haas M, Vlcek V, Balabanov P, et al. European medicines Agency review of ataluren for the treatment of ambulant patients aged 5 years and older with Duchenne muscular dystrophy resulting from a nonsense mutation in the dystrophin gene. Neuromuscular Disorders 2015;25:5-13.

14. Flanigan KM. Duchenne and Becker muscular dystrophies. Neurol Clin 2014;32:671-88.

15. Howard MT, Shirts BH, Petros LM, et al. Sequence specificity of aminoglycoside-induced stop codon readthrough: potential implications for treatment of Duchenne muscular dystrophy. Ann Neurol 2000;48:164-9 http://www.ncbi.nlm.nih.gov/pubmed/ 10939566

16. Siddiqui N, Sonenberg N. Proposing a mechanism of action for ataluren. Proc Natl Acad Sci U S A 2016;113:12353-5.

17. Niks EH, Aartsma-Rus A. Exon skipping: a first in class strategy for Duchenne muscular dystrophy. Expert Opin Biol Ther 2017;17:225-36.

18. Consalvi S, Saccone V, Mozzetta C. Histone deacetylase inhibitors: a potential epigenetic treatment for Duchenne muscular dystrophy. Epigenomics 2014;6:547-60.

19. Mishra MK, Loro E, Sengupta K, et al. Functional improvement of dystrophic muscle by repression of utrophin: let-7c interaction. PLoS One 2017;12:e0182676.

20. Aslesh T, Maruyama R, Yokota T. Skipping multiple exons to treat DMD-Promises and challenges. Biomedicines 2018;6.

21. Govoni A, Magri F, Brajkovic S, et al. Ongoing therapeutic trials and outcome measures for Duchenne muscular dystrophy. Cell. Mol. Life Sci. 2013;70:4585-602.

22. Gee $P, X u H$, Hotta A. Cellular reprogramming, genome editing, and alternative CRISPR Cas9 technologies for precise gene therapy of Duchenne muscular dystrophy. Stem Cells Int 2017;2017:1-11.

23. Hagan M, Ashraf M, Kim I-M, et al. Effective regeneration of dystrophic muscle using autologous iPSC-derived progenitors with CRISPR-Cas9 mediated precise correction. Med Hypotheses 2018;110:97-100.

24. Moher D, Liberati A, Tetzlaff J, et al. Preferred reporting items for systematic reviews and meta-analyses: the PRISMA statement. PLoS Med 2009;6:e1000097.

25. Hamuro L, Chan P, Tirucherai G, et al. Developing a natural history progression model for Duchenne muscular dystrophy using the Six-Minute walk test. CPT Pharmacometrics Syst. Pharmacol. 2017;6:596-603.

26. Nadarajah VD, van Putten M, Chaouch A, et al. Serum matrix metalloproteinase-9 (MMP-9) as a biomarker for monitoring disease progression in Duchenne muscular dystrophy (DMD). Neuromuscular Disorders 2011;21:569-78.

27. MCDonald CM, Henricson EK, Han JJ, et al. The 6-minute walk test in Duchenne/Becker muscular dystrophy: longitudinal observations. Muscle Nerve 2010;42:966-74.

28. Echigoya Y, Lim KRQ, Trieu N, et al. Quantitative antisense screening and optimization for exon 51 skipping in Duchenne muscular dystrophy. Molecular Therapy 2017;25:2561-72. 